\title{
VOLUNTEER GEOGRAPHIC INFORMATION IN AFRICA
}

\author{
Aster Denekew Yilma \\ United Nations Economic Commission for Africa - denekewa@,un.org
}

\begin{abstract}
KEY WORDS: Volunteer Geographic Information; VGI; Crowd sourcing; Community Mapping; Africa
\end{abstract}
\begin{abstract}
:
During the past few years much effort has been put into developing community-based methods to capture and analyze a large amount of data in a systematic manner. This new source of geo-referenced data could be very important throughout Africa where crowd sourcing activities or volunteered geographic information (VGI) enhance participation and decision making based on local knowledge. VGI has the potential to be a significant source of information for a variety of applications. It will especially help national mapping authorities to build and update their national geospatial datasets, and improve the availability and currency of the countries fundamental and thematic datasets. Governments therefore need to take proactive measures to develop strategies maximizing on the opportunity in the current technological development. This calls out the need to developing strategic guidance on how to strengthen the communities' participation in data collection.

The United Nations Economic commission for Africa developed a document on the best practices and principles for adopting community mapping and VGI in the mapping practices in Africa. An expert group meeting is held to gather experts in the field to discuss on the issues, review best practices and current status on citizen participation in community mapping and to develop a roadmap for the adoption of the technology and agree on the guiding principles. The outcome of the meeting resulted in the framing of guiding principles for community mapping which is published in 2017.
\end{abstract}

This paper therefore provides overview of VGI in Africa and the efforts made to date.

\section{BACKGROUND}

\subsection{Volunteer Geographic Information}

One of the most important aspects in Web 2.0 development is the emergence of crowd sourced information (Hakley, 2010). Crowd sourcing is referred as how large group of users are performing functions that were difficult to automate or expensive to implement (Hakley, 2010). (Hakley, 2010) also explained that the crowd sourcing can be used in large scale community activities that were difficult to implement before Web 2.0. Such community activities recently focused on the collection of information. One of the successful crowd sourcing examples is wikipedia, the free encyclopedia. Wikipedia is written collaboratively by largely anonymous volunteers.

The potential of crowd sourcing activity for geographic information has also captured the attention of researchers in the geospatial field. Goodchild described this activity as Volunteer Geographic Information (VGI) (Goodchild, 2007) and (Hakley, 2010). Information created by citizens is generally called usergenerated content (UGC), and specifically the geographic information is VGI. VGI is digital spatial data collected voluntarily by citizens rather than by formal data producers (Goodchild, 2007) and (Fu and Sun, 2011). (Goodchild, 2007), described user-generated content on the web, as: "a remarkable phenomenon ... the widespread engagement of large numbers of private citizens, often with little in the way of formal qualifications, in the creation of geographic information, a function that for centuries has been reserved to official agencies.
... I term this volunteered geographic information (VGI), a special case of the more general Web phenomenon of usergenerated content ..."

The Web 2.0 technology, which enables the user to create its own content and to edit content created by others, is the technology behind the UGC, and so the VGI. The Web 1.0 technology is one directional, where users only retrieve or download the information from the web pages. Contrary to web 1.0 , web 2.0 technology is bi-directional, allowing the users to collaborate and share the common information with each other via the internet. Thus, users of VGI could upload their own geographic information to web server, download the geographic information shared by others, and even edit the geographic information created by others (Jia, 2010).

In the past few years there has been rapid growth of interest in VGI (Goodchild, 2007). It has proven successful as a means of acquiring timely and detailed geographic information at very low cost (Goodchild and Li, 2012). With the development of the Web 2.0 and mobile devices improvement to provide data related to their location, people began to be more involved in VGI, providing data and information that are in many cases more detailed and of a higher quality than that provided by official institutions (Horita et al., 2013). The way geographic data are collected at an individual basis is different from the traditional way by the national mapping agency. The use of GPS allows untrained users to automatically acquire their location accurately, facilitating gathering high accuracy geographic information. GPS can be accessed by different products such as camera and mobile 
devices including mobile phones so that the information collected with the devices will be automatically tagged with the geographic coordinates. So far, a huge amount of geographic data has been collected due to the increasing number of contributors and volunteers.

\subsection{Types of VGIs}

The last decade has seen a revolutionary development where many service providers have started providing global and local maps - including and most notably Google Inc. In some applications maps can be worked on by individuals, collectives and communities, enabling them to place information on base maps and utilize the power of mapping technologies for analysis and presentation. For instance, the Google Maps API is a way to organize information on maps, both manually and programmatically. Google Earth is also used for innovative community mapping with significant utility for civil society.

By 2004, it had become evident that individuals could create their own digital geographic information in the form of high-quality, on-line maps, essentially at no cost. OpenStreetMap became the well-known and successful of a number of efforts begun at that time. Websites such as Wikimapia and OpenStretMap use Webbased "crowd-sourcing" technologies, which outsource tasks to the large group of Web users to collect data, identify problems, and invite solutions to problems through the use of VGI (Fu and Sun, 2011).

Collaborative Web-based efforts like OpenStreetMap, Google map maker, etc. enable experts and amateur enthusiasts to create and share limited, theme oriented geospatial information.

Wide range of VGI efforts have taken place in recent years. Few examples of successful VGI are:

OpenStreetMap (OSM) (https://www.openstreetmap.org/): OpenStreetMap aims to map data that are free to use, editable and licensed under new copy right scheme, the creative commons, to enable free access to current digital geographic information across the world (Hakley, 2010). By relying on VGI contributions, OSM is able to develop a map product that competes with commercial maps.

In the study conducted by (Hackley, 2010), the comparisons between OSM and Ordnance Survey road maps showed that VGI can reach very good spatial data quality.

Google Map Maker (https://www.google.com/maps/): Google launched mapping applications with collaborative features, most importantly Google Maps with the Google Map Maker feature. Tools such as Google Map Maker have documented an enormous mass of overlay information on the base maps today contributed by individuals.

Wikimapia (www.wikimapia.org/): Wikimapia is open content collaborative map where anyone can create place tags, and share knowledge. It is a project to "describe the whole world" by identifying and providing detailed descriptions of point or area features. Wikimapia is in effect a crowd-sourced gazetteer (Goodchild and Hill, 2008), a catalog of place names, geographic locations and feature descriptions. Wikimapia adapts some of the successful procedures that have been successful in Wikipedia and applied to the creation of gazetteers. Anyone with internet connection can select an area and describe it, can edit features, and volunteer reviewers monitor the result by checking for accuracy and significance (Goodchild, 2007).

VGI is classified in three types based on the motivation of contributors as: market driven, social networking and civil / government. Examples are given in these different classes.

1.2.1 Market driven VGI: Firms like TeleAtlas, Navteq and TomTom used Web-based customer input to locate and qualify mapping errors and/or feature updates required in their road network databases (Biersdorfer, 2007) and (Coleman et al., 2009).

For example, TomTom is a company which design and develop innovative navigation devices, navigation and mapping products, as well as state-of-the-art fleet management solutions and location-based products. As a commercial company TomTom uses the location data collected by the users, with the permission of the user. The community is producing location information and traffic information while using the portable navigation devices which again is used by TomTom to improve the service for the community of users by updating maps, providing real-time traffic information etc. This form of community data collection or VGI is again benefiting the whole community of the product users.

1.2.2 Social Networking VGI: GeoTweeting is one example of social media VGI, launched by Twitter. GeoTweet has feature that facilitates VGI, adds map links to tweets with optional photos and notes. It is also an example of VGI that can be used for disaster and emergency situations. It is used for monitoring and reporting activities, incidents, or events in real time, reported by users at their locations.

1.2.3 Civic/Government type VGI: One example of civic VGI is Ushahidi (http://www.ushahidi.com/). Ushahidi is a global non-profit technology company that specializes in developing free and open source software for information collection, visualization and interactive mapping. Ushahidi originates from Kenya initially developing the Ushadidi platform to map incidents of violence in Kenya and peace efforts throughout the country after the postelection fallout at the beginning of 2008, based on reports submitted via the web and mobile phones. The Ushahidi platform is built as a tool to easily crowdsource information using multiple channels, including SMS, email, Twitter and the web. The crowdmap application is hosted in the Ushahidi platform to crowdsource information, and is applied to different disaster situations to measure the impacts of disaster.

\section{VGI TRENDS IN AFRICA}

Besides Web 2.0 technology which created the possibilities of user generated content, new mobile technologies also brought what was once in the domain of the GIS experts into mainstream society. Location based applications and services on these mobile devices is changing our world into one in which any citizen with GPS enabled phone can become a geo-sensor and collect data and attributes on a particular location and more importantly broadcast this information to the world voluntarily using the internet and 
social media. This crowd-sourcing innovation is considered to be the future trend in geospatial industry development.

Recently, African countries are also following the global trend in harnessing the information contributed by citizens, specifically VGI. There are a number of cases where African countries have implemented VGI projects and local governments showed interest towards the adoption of crowdsourced information.

\subsection{Best practices of VGI in Africa}

Few of the examples in implementations of VGI projects, from the study conducted by (Hakley et al., 2014) and compiled as a report to the Global Facility for Disaster Reduction and Recovery (GFDRR), World Bank, are presented below.

Kenya: Map Kibera project was launched in 2009 with the aim to map the unmapped Kibera by actively participating the local people. The process involves collecting GPS tracks and tracing them in the OSM platform. The project also offered an opportunity for mappers to enhance points of interest such as water points, schools, public toilets, police stations, and clinics. The project gained acceptance by the local government.

South Sudan: The aim of the project was to engage volunteers Sudanese Diaspora to map the poorly mapped South Sudan so that the infrastructure and the economy of the country could be developed. The project was launched in 2011 implemented by Google with the support of World Bank, UNOSAT and RCMRD. The process involves satellite imagery of the region uploaded to Google Earth and maps for editing and updating on Google maps using Google Map Maker. Citizens mapped using available web tools and their local knowledge and after approval the map is made available in Google to all users worldwide. The project also gained acceptance by local government and international organizations. The mapping was used by the Satellite Sentinel project of the Enough Project, Digital Globe, Not On Our Watch, UNITAR, UNOSAT, the Harvard Humanitarian Initiative, and Trellon.

South Africa: The iCitizen project in South Africa was launched with the aim to improve the life of citizens by collecting reports on fundamental problems with infrastructure and services and passing them to the relevant authority for resolution. The process involves reporting of issues by the public forwarding geo-tagged photographs, sending in locations via SMS, or reporting issues via email.

Uganda: The Community Knowledge Workers (CKW) initiative in Uganda aims to train local people in the use of technology for data collection and sharing farming knowledge to improve their farming efficiency and as a result their livelihoods. Participants of $\mathrm{CKW}$ use their mobile phones to collect information in livestock and farming. The data collection takes place offline using the GPS sensor of the mobile device and properly designed forms. Once the phones are in areas of wireless or mobile network the data are transferred to the central server and shared to other farmers and users. The information shared in turn help farmers and policy makers to address the needs of farmers and provide market price updates, receive weather forecasts, and access potential disease outbreak, etc.
Somalia: UNHCR, DigitalGlobe, Tomnod, Standby Task Force (SBTF), and Ushahidi collaborated in a humanitarian project to geo-locate and map all shelters in Somalia's Afgooye corridor with the aid of satellite imagery provided by the Standby Volunteer Task Force. The goal of the project was to test the feasibility of crowd sourcing rapid shelter enumerations of internally displaced persons to support population estimates.

\subsection{Emerging issues in VGI}

Like any other new research field, VGI face issues or common problems to be addressed or further researched. These issues include the data discovery and knowledge discovery, data uncertainty or accuracy, human privacy or liability, the motivation for VGI, etc. (Jia, 2010).

Data discovery will be an issue, as the big data coming from the VGI community exponentially increases, accessing spatial data and identifying its usability might be a problem.

Data quality and uncertainty is also a concern in VGI. In most cases, the spatial data collected by volunteer does not go through formal quality control procedures. The quality of VGI also depends on the source data used to produce it as well as the knowledge of the contributor. Data contributed by amateur could be less accurate than the professional user.

The threat to the national authorities to adapt to the paradigm shift is another issue in implementing VGI technology. As (Coleman et al., 2009) puts it, the potential exists for government mapping agencies to harness the power of new media and voluntarism in order to improve their own change detection and geospatial data updating processes. It has the power to complement existing practices and enable new production systems. However, these certainly bring about tensions and modifications to cultures, policies and processes necessary for established public sector organizations to accommodate volunteered information to their databases.

If national mapping organizations wish to tap into the distributed knowledge, time and energy of volunteer producers to contribute authoritative geospatial data, they must be prepared to entertain some important procedural and cultural changes that build on the motivations and recognize the characteristics of the culture.

If a mapping organization wishes to capitalize on a distributed network of volunteer geospatial data producers (a concept used to define the volunteers who collect data/information voluntarily as data producer and also use volunteered information as consumers or users), then it must start refocusing attention across what happens both inside that organization and also in the new social network of geo-information production. New rules and standards will be required to take into account the values of these volunteers - equity, security, community building, privacy — in evaluating the performance of this new production system (Coleman et al., 2009).

The report of the World Bank by (Hakley et al., 2014) also highlighted that VGI should not be seen as an activity that will replace the work of professionals but as one that enhances it. However, in most cases, VGI is perceived as a challenge to existing procedures and professional standing which leads to 
negative response. There is also a need to integrate VGI processes including issues of engagement and feedback to contributors in to established systems, practices and procedures. The study also indicated that adopting crowd sourcing will require additional resources to manage the crowd sourcing process, the data collected and engaging with the communities involved. Therefore, governments should consider long term plan and assess the sustainability of their adoption of crowd sourcing.

\section{VGI APPLICATIONS}

There are a number of applications which can benefit from the volunteer geographic information. Some of the applications which are considered important and are of best practice are mentioned here.

\subsection{Updating Fundamental Data Sets}

The utilization of VGI for spatial information collection and updating is now widely used by OpenStreetMap, TeleAtlas, NAVTEQ and Google Maps. Government organizations are also recently employing volunteered input into their mapping programs. Government organizations have now also realized the power of VGI and crowd sourcing and are interested in utilizing these technologies for Spatial Data Infrastructure development (Paudyal, et al., 2012).

National mapping agencies can use, for example, Google maps and OpenStreetMap to update their fundamental datasets. Road Map as one of the framework datasets can be updated using these maps.

(Coleman, 2010) said authoritative mapping organizations should extend these services to permit incremental updates to existing databases. (Coleman, 2010) also gave examples, where State governments in Victoria, Australia and North-Rhine Westphalia, Germany employ volunteered input to their mapping programs.

\subsection{Statistics}

Data collection is facilitated by using new technologies such as the use of mobile devices and digital map applications such as Google maps, and the web 2.0 technologies. Volunteers can collect data/statistics in different theme and sector including their location. VGI is one of the new technologies which can be tapped to collect statistical information by volunteers and citizens.

\subsection{Thematic Applications}

3.3.1. Natural Resource Management: A study was conducted by (Paudyal, et al., 2012) to understand the use of VGI in the area of catchment management in Australia. This study identified the major community driven or volunteer initiatives which spread over all the themes such as land, water, biodiversity, coast, and marine for catchment management. The major ones from these volunteer activities include: The Landcare, Waterwatch, Coastcare, Land for wildlife, Birdwatch, and Vegewatch. (Paudyal et al., 2012) concluded the level of applicability of open source products and VGI to various catchment management issues is very high.
3.3.2. Biodiversity: VGI is also used in biodiversity studies to collect data on biodiversity, georeference that information, display and disseminate the information. VGI in biodiversity studies involves an array of data gathering, from the compilation of data on species occurrences to information on species abundances, all collected by volunteers.

Data gathered by volunteers has been prominent for collecting plants species and for birds count, as well as breeding bird surveys. VGI plays a critical role for monitoring biodiversity in the face of increasing species extinctions, and can provide substantial support for biodiversity research. There are several important VGI initiatives that are ongoing today that provide critical information that may be used by researchers. These include: eBird, Audubon Christmas Bird Count, Breeding Bird Survey, E-Flora and E-Fauna (Klinkenberg, 2015).

3.3.3. Tracking: VGI can be used for tracking and fleet management in emergency situation. Since most people now use GPS enabled smart phones these can be utilized in an emergency. A VGI based fleet tracking solution adds a tremendous capability in an emergency situation. Supervisors and other emergency workers can not only know where the official responders are but they would also know the locations of the volunteers. This would make the task of coordinating their efforts much easier and would keep them informed as to the progress of both responders and volunteers (Hamilton and Janowicz, 2014).

(Hamilton and Janowicz, 2014) presented cases of three crises situations in Florida; flooding, tornado and hurricane. By using volunteer geographic services, the progress of the volunteers is constantly monitored since their GPS-enabled smart phones periodically update their location. This allows for the supervisory staff to more efficiently route the volunteers and to track their progress towards their destinations. Thus the residents in the flooded area know that assistance is not only on the way but they know approximately when it is due to arrive and what is coming.

3.3.4. Disaster Management/Emergency Situation: VGI is becoming especially useful during emergency where responders are not in a position to access critical data in real time. Emergency responders have limited staff and ability to acquire and synthesize the geographic information that is vital to effective response. In many cases people living far from the affected area have been better informed through the media than those managing and carrying out the relief effort (Goodchild and Glennon, 2010). Affected areas often lose power, Internet connection, impeding access to relevant information and computing capabilities. Today, volunteers have mobile devices equipped with digital cameras, GPS, digital maps and numerous other resources and they are in a position to contribute during such emergency situations. Moreover, citizens are able to access and interpret data streams from satellites with comparatively fine temporal and spatial resolution. They can compile this information and synthesize it in the form of maps, using services such as Google Maps, and provide easily accessed and understood disaster situation reports (Goodchild and Glennon, 2010).

(Goodchild, 2011) also stressed in his keynote speech in a VGI workshop, the importance of time in VGI to create an accurate picture of what's occurring and when in a particular situation. He also stated that VGI can readily claim to be the most current data 
source however it has been criticized for having poorer positional accuracy and overall veracity. He countered this, citing Linus's Law, which states that the more people involved and watching over a project, the more likely errors can be spotted and fixed quickly. He further stressed the contributions of citizens will tend to be more accurate for places that they live in and knows best, quoting the Tobler's Law, which states, "Everything is related to everything else, but near things are more related than distant things."

(Goodchild, 2011) in his keynote speech also said that "The most compelling case for VGI is during emergencies", in the event that experts being sometimes scarce on the ground, it's important to turn to citizens who can contribute data through social media or other means, in the context of relying on the currency and coverage.

A good example of VGI in emergency situation is the responses to Haiti Earth quake, in 2010. OpenStreetMap (OSM) project volunteers working outside Haiti created a digital street map of Port-au-Prince and other places in Haiti very rapidly using fineresolution imagery to trace vector maps of streets and other features. The Ushahidi Project was also supporting the response, by posting appeals for help, providing translation from Creole (local language) into English by another group of online volunteers. Together, these VGI projects were instrumental in guiding first responders to disaster victims. The International Network of Crisis Mappers (also an Ushahidi collaborator), has established the Standby Volunteer Task Force: An Online Community for Live Crisis Mapping. Crisis Mappers also helped the Haiti earthquake disaster response in 2010 by organizing the digital response to the disaster.

\section{EFFORTS ON VGI AT CONTINENTAL LEVEL}

During the past few years much effort has been put into developing community-based methods to capture and analyze a large amount of data in a systematic manner. This new source of geo-referenced data could be very important throughout Africa where crowd sourcing activities or volunteered geographic information participating the citizen are best positioned to support better understanding of local issues and therefore the development of decision support strategies and mechanisms. National Mapping Authorities should therefore consider this potential source of data to supplement and improve the coverage of their national mapping. Governments need to take proactive measures to develop strategies maximizing on the opportunity in the current technological development. This calls out the need to give close attention to developing strategic guidance on how to strengthen the communities' participation in data collection.

As a first step, United Nations Economic Commission for Africa (ECA) conducted a Workshop on Community mapping in March 2013 to introduce the concept of Community Mapping, Crowdsourcing and VGI, to discuss the methodology for mainstreaming VGI into national mapping programmes, the challenges and issues as well as the way forward to developing guidelines.

As a follow up activity ECA developed a document on the best practices and principles for adopting the community mapping in the mapping practices in Africa. This background document was intended for discussion and served as a stepping stone to the development of the guidelines. An expert group meeting is therefore organized to gather experts in the field to discuss on the issues and to develop a roadmap for the adoption of the technology and agree on the guiding principles. The outcomes of the meeting were used in framing the guideline document which is published in August 2017 (UNECA, 2017). This guideline document outlines some of the policy principles and strategic options. It stressed that the national mapping agencies have the mandate to produce authoritative fundamental geospatial data sets and need to be re-energized through capacity development in order to find innovative ways of achieving their mandates, generating more geospatial information using VGI. The document also outlined some of the legal issues that hinder effective implementation of VGI in Africa including National security policies and laws, IP laws and policies, lack of or restrictive regulation of data collection methods e.g. Use of UAV. It also identifies the Policy options present to effectively harness the potentials of VGI in Africa, such as open data supported by governments, promotion of access to information and open standards, and integration of VGI into the NSDI development.

\section{CONCLUSION}

VGI has the potential to be a significant source of information for a variety of applications, including real-time disaster monitoring and early warning. According to (Goodchild and Li, 2012), VGI has enormous advantages: it is free, can be timely and can provide types of data not previously utilized in mapping practice. There would be great benefit if its quality could be improved and assured.

This new source of geospatial data will be important for Africa where community mapping activities enhance participation and decision making based on local knowledge. This will especially help national mapping authorities to build and update their national geospatial datasets, and improve the availability and currency of the countries fundamental and thematic dataset.

\section{REFERENCES}

Biersdorfer, J. D., 2007. Updating Maps on the Spot and Sharing the Fixes. New York Times article, 14 June, 2007, http://www.nytimes.com/2007/06/14/technology/14gps.html (1 July 2015).

Coleman, D. J., 2010. Volunteered Geographic Information in Spatial Data Infrastructure: An Early Look At Opportunities and Constraints. Department of Geodesy and Geomatics Engineering, University of New Brunswick.

Coleman, D. J., Georgiadou, Y., and Labonte, J., 2009. Volunteered Geographic Information: the nature and motivation of produsers. Article under Review for the International Journal of Spatial Data Infrastructures Research, Special Issue GSDI-11, submitted 2009-03-27.

$\mathrm{Fu}, \mathrm{P}$., and Sun, J., 2011. Web GIS: Principles and Applications. 2011, ESRI Press,: Redlands, California, USA

Goodchild, M.F. and Li, L., 2012. Assuring the quality of volunteered geographic information, Center for Spatial Studies 
and Department of Geography, University of California, Santa Barbara, USA, Elsevier, 2012.

Goodchild, M. F., 2011. It's About Time. ESRI ArcWatch, March 2011, http://www.esri.com/news/arcwatch/0311/power-ofvgi.html ; (1 August 2015).

Goodchild, M.F. and Glennon, J. A., 2010. Crowdsourcing geographic information for disaster response: a research frontier. 2010, Taylor \& Francis, UK.

Goodchild, M.F. and Hill, L.L., 2008. Introduction to digital gazetteer research. International Journal of Geographical Information Science. 22(10): 1039-1044.

Goodchild, M.F., 2007. Citizens as Censors: the World of Volunteered Geography. Geojournal 2007 69:211-221, Published online November 2007, Springer Science+Business Media B.V. 2007.

Hakley, M., Antoniou, V., Basiouka, S., Soden, R., and Mooney, P., 2014. Crowdsourced Geographic Information use in Government. Report to Global Facility for Disaster Reduction and Recovery (GFDRR), World Bank.

Hakley, M., (2010), How good is Volunteered Geographic Information? A comparative Study of OpenStreetMap and Ordnance Survey datasets. Environment and Planning B: Planning and Design 2010, volume 37, pages 682- 703.

Hamilton, D., and Janowicz, K., 2014. Volunteered Geographic Services: The Case of Fleet Management. April 2014, https://gis.e-

education.psu.edu/sites/default/files/capstone/Hamilton_paper 59 6B 20140401.pdf (31 August 2015).

Horita, F., Degrossi, L.C., Assis, L.F.F.G., Zipf, A., Porto de Albuquerque, J., 2013. The use of Volunteered Geographic Information and Crowdsourcing in Disaster Management: a Systematic Literature Review, Proceedings of the Nineteenth Americas Conference on Information Systems, Chicago, Illinois, August 15-17, 2013.

Jia, T., 2012. Geospatial Knowledge Discovery using Volunteered Geographic Information: a Complex System Perspective. Doctoral Dissertation, 2012, ISBN 978-91-7501531-6, Division of Geodesy and Geoinformatics, Department of Urban Planning and Environment, Royal Institute of Technology (KTH), Sweden.

Jia, T., 2010. Exploring Massive Volunteered Geographic Information for Geographic Knowledge Discovery. December 2010, Thesis, Royal Institute of Technology, Sweden.

Klinkenberg, B., 2015. Citizen Science And Volunteered Geographic Information: Can These Help in Biodiversity Studies. Department of Geography, University of British Columbia, http://ibis.geog.ubc.ca/biodiversity/VGI--

VolunteerGeographicInformation.html (10 August 2015).

Paudyal, D.R., McDougall, K., and Apan, A., 2012. Exploring the Application of Volunteered Geographic Information to Catchment Management: A Survey Approach. ISPRS Annals of the Photogrammetry, Remote Sensing and Spatial Information Sciences, Volume I-4, 2012, XXII ISPRS Congress, 25 August 01 September 2012, Melbourne, Australia.
UNECA, 2017. Volunteered Geographic Information in Africa, United Nations Economic Commission for Africa (UNECA), Addis Ababa, Ethiopia, 2017. 